\title{
Coronary artery-right ventricular fistula after endomyocardial biopsy
}

\author{
T J LOCKE, S S FURNISS, C G A MCGREGOR \\ From the Departments of Cardiothoracic Surgery and Cardiology, Freeman Hospital, Newcastle upon Tyne
}

SUMMARY A coronary artery-right ventricular fistula developed after endomyocardial biopsy in a recipient of an orthotopic cardiac transplant.

The technique of percutaneous transvenous endomyocardial biopsy of orthotopic cardiac grafts was developed in 1971 in the laboratory and was clinically applied the next year at Stanford University. ${ }^{1}$ Biopsy is performed by cannulation of the right internal jugular vein and passage of the biopsy forceps through a sheath into the right ventricle under fluoroscopic control. Because it is a safe procedure endomyocardial biopsy can be performed as often as indicated, and this permits the diagnosis of rejection before graft damage becomes irreversible.

\section{Case report}

A 44 year old women underwent orthotopic cardiac transplantation for congestive cardiomyopathy in August 1986. Appendícectomy for acute appendicitis was performed on the tenth postoperative day. She was discharged on the twenty second postoperative day and remained symptom free until October 1986. Then she became breathless on exertion, and orthopnoea and ankle swelling developed. On examination she had a raised jugular venous impulse, hepatomegaly, ankle oedema, and a third heart sound. Chest $x$ ray showed bilateral pleural effusions. An endomyocardial biopsy specimen showed moderate rejection with myocyte necrosis. She was treated with a rapidly tapering dose of oral prednisolone. Follow up endomyocardial biopsy specimens confirmed the resolution of this episode of rejection but her congestive cardiac failure did not. improve. Investigation including echocardiography was suggestive of either pericardial constriction or myocardial restriction.

Requests for reprints to Mr C G A McGregor, FRCS, Department of Cardiothoracic Surgery, Freeman Hospital, Newcastle upon Tyne NE7 7DN.
The myocardium seemed to be abnormally echogenic and there was global left ventricular dysfunction. In December 1986 cardiac catheterisation confirmed the presence of pericardial fluid and a constrictive/restrictive pattern with reduced ventricular function.

Selective injection of the left coronary artery showed dye entering the right ventricle (figure).

A decision was made to retransplant the patient. Her degree of heart failure worsened while she was waiting for a suitable donor. By the time of emergency retransplantation in February 1987 legionella peritonitis and pneumonia had developed. She died of cerebral haemorrhage secondary to disseminated intravascular coagulation caused by the uncontrolled septicaemia. Postmortem injection of the coronary arteries was not performed and the fistula was not demonstrated in the excised heart.

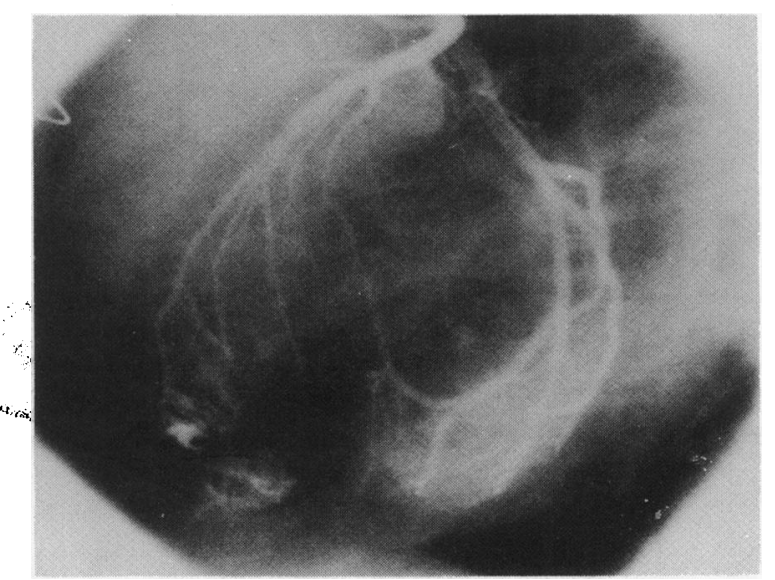

Figure Selective injection of left coronary artery showing leakage of dye from the distal vessel into the right ventricular cavity. 
82

\section{Discussion}

Endomyocardial biopsy remains the reference standard for the diagnosis of cardiac allograft rejection. Although much effort has been directed to the search for non-invasive methods of diagnosis of rejection, none has yet replaced histological examination of biopsy material. With care, the risk of endomyocardial biopsy to the patient is minimal.

At Stanford University, 10000 endomyocardial biopsies have been performed with no mortality. Cardiac perforation has developed in three of the heart transplant recipients from whom biopsy specimens have been taken (M E Billingham, personal communication).

Our case represents a previously unreported complication of endomyocardial biopsy. In the Freeman Hospital 400 biopsies have been performed in 28
Locke, Furniss, McGregor

cardiac transplant recipients. This is the only case of $\frac{\text { I }}{\mathbb{D}}$ morbidity associated with biopsy. The fistula was not clinically important and was asymptomatic. The fistula must have been created when a biopsy specimen was taken from the junction of the right ventricular free wall and the septum-the point at which the left anterior descending artery lies closest to the right ventricular cavity. This report emphas $-\overline{\mathrm{D}}$ ises that the biopsy specimens of the endomyocar- 0 dium should not be taken from the apex of the rightes ventricle.

\section{Reference}

1 Caves PK, Stinson EB, Billingham ME, Rider AK Shumway NE. Diagnosis of human cardiac allograft rejection by serial cardiac biopsy. J Thorac Carjo diovasc Surg 1973;66:461-6. 